\title{
Analysis of complex inflatable structures using a multi-body dynamics approach
}

To be presented at the 49th AIAA/ASME/ASCE/AHS/ASC Structures, Structural Dynamics, and Materials Conference, Schaumburg, IL, USA.

\author{
J. Breukels ${ }^{1}$ and W.J. Ockels ${ }^{2}$ \\ Delft University of Technology.
}

\begin{abstract}
[Abstract] This paper outlines a simulation of inflatable structures through the use of multi-body dynamics. Beams are approximated by rigid elements, connected by spherical joints and 3-dimensional torque vectors. The stiffness of the torque vector determines the bending behavior of the beam. The stiffness is a variable stiffness with deflection. It is dependent on internal pressure and beam radius. By varying the stiffness of the torsion springs, the nonlinear behavior of an inflatable structure can be simulated. Functions for the stiffness of the torsion springs are obtained by matching to existing measured data. The simulation uses these functions to simulate more complex structures.
\end{abstract}

\section{Nomenclature}

$\mathrm{p}=$ Internal overpressure

$\mathrm{r}=$ Beam radius

$\mathrm{t}=$ Membrane thickness

$\sigma_{1}=$ Stress in longitudinal direction

$\sigma_{\mathrm{c}}=$ Stress in circumferential direction

\section{Introduction}

Mechanics of inflatable structures has been the subject of extensive research. Especially with the coming of stronger and lighter membrane materials, there has been a great deal of interest in inflatable structures to be used in a number of applications. The theory of the mechanics of inflatable structures is rather complex due to its non-linear behaviour. Once wrinkling sets in, the bending behaviour of the structure becomes far more complex due to the fact that, as a result of bending, local wrinkling has a large impact on the behaviour of the entire structure.

This generally leads to complex finite element analysis which take a lot of time to set up and to process. This can be a costly endeavour. Furthermore, from a designer's point of view, the long delays due to FEM processing can be detrimental to the final product. As a designer, one would like to have quick feedback as to the behaviour of the structure. This is especially true in the field of kites, boats or other tubular structures. In these structures, one is not necessarily interested in exactly what location wrinkling will occur at exactly what amount of torque or bending moment. As a matter of fact, the conventional finite element analysis creates an overshoot of details which are not needed in these design disciplines.

Up until now the design of kites has been done empirically. New kites are designed at such fast pace, that a complex finite element analysis is simply not practical. Furthermore, the excess of data created by such an analysis is not of any essential use. In this design discipline, one would like to have a tool which may be somewhat less accurate, but gives results almost instantly. This paper presents a method for the analysis of complex inflatable structures such as kites or other tubular structures using a multi-body dynamics approach.

\section{Mechanical behavior of inflatable structures}

Veldman [Ref 17] stated that inflatable structures are part of a characteristic group called tensile structures. A tensile structure is a membrane like structure that requires tensile pre-stresses in order to bear externally applied

1. PhD Student, Faculty of Aerospace Engineering, Kluyverweg 1, 2629HS, Delft, the Netherlands

2. Professor and chair holder, Faculty of Aerospace Engineering, Kluyverweg 1, 2629HS, Delft, the Netherlands 
loads. An inflatable structure creates these pre-stresses by means of pressure differential over the skin. The amount of pre-stress, which translates into the amount of internal pressure, determines the load-bearing behavior of the structure. In this paper, the focus is on tubular structures. Many different wings and support structures can be achieved by using a tubular structure as a basis. For kites, tubular structures are being used in conventional tubekites which are one of the most commonly used kites in the kite-surfing world. Their rigidity enables the kite to maintain its shape. Inflatable sail wings use tubular structures as well. The kiteplane design [REF 3] is an example of a tubular inflatable structure.

For pressurized tubes, the stresses in the membrane are governed by the boiler formulas:

$$
\begin{gathered}
\sigma_{l}=\frac{p r}{2 t} \\
\sigma_{r}=\frac{p r}{t}
\end{gathered}
$$

For a conventional hollow, circular beam in bending, the stresses are given by:

$$
\sigma=\frac{M r}{\pi r^{3} t}
$$

In an inflatable beam, the tensile pre-stress and the compressive stress due to bending play a prominent role. The inflatable beam is considered a membrane structure. This means that its material cannot sustain any compressive stress. By creating a tensile pre-stress, the structure as a whole obtains bending rigidity due to the fact that the sum of the tensile pre-stress and the compressive stress due to bending remains positive.

An inflatable beam under a load can exist in three distinct states [Ref 8]. In the state, the sum of the tensile pre-stress and the stress due to bending is positive everywhere in the skin of the beam. This state is called "the unwrinkled state" and it is the state in which the beam exhibits the most rigidity. In the unwrinkled state, the bending behavior is linear, assuming the young's modulus is constant (isotropic material).

The second state is called "the wrinkled state". In this state, somewhere in the beam the sum of the tensile pre-stress and the bending stress has become negative. The membrane material cannot sustain a negative stress and therefore, it starts to wrinkle. In the case of a cantilever beam loaded by a positive bending moment (bending the tip down), the compressive stress due to the bending is the greatest at the root on the lowest side of the beam. It is here where wrinkling will occur. Wrinkling generally occurs first in the location which has the highest compressive stress due to the externally applied load. A beam in the wrinkled state will exhibit a lower bending stiffness. Furthermore, its bending behavior will no longer be linear. As the externally applied load grows in magnitude, the wrinkled area will grow in both longitudinal and circumferential direction. The larger the wrinkled area, the less bending stiffness the beam will exhibit.

As the wrinkled area grows under a growing externally applied load, the wrinkled area will, at one point, pass the neutral line in circumferential direction. In this situation, there is a cross-section where the entire area under the neutral line is no longer supporting any compressive loads. This signals the third state of the inflatable beam called "the collapsed state" Where the beam is no longer able to bear the externally applied load and collapses. The bending rigidity is reduced to nearly zero.

Figure 1 shows the three states of an inflatable structure and indicates the rigidity of the different states. One might be inclined to say that a structure should never venture into the wrinkled state. For metal structures, one would never allow wrinkles to occur due to the detrimental effects of plastic deformation to the material. The wrinkled state in an inflatable structure, however, is completely reversible. And even though wrinkles exist, the structure is still able to bear a considerable amount of externally applied loads. The wrinkled state comes with a penalty of increased flexibility. But such a penalty is not necessarily a problem. For instance, an increased flexibility allows a wing to 
deal with wind gusts by flexing its structure instead of displacing its entire body. Therefore, the wrinkled state is a perfectly acceptable state for an inflatable structure to exist in.

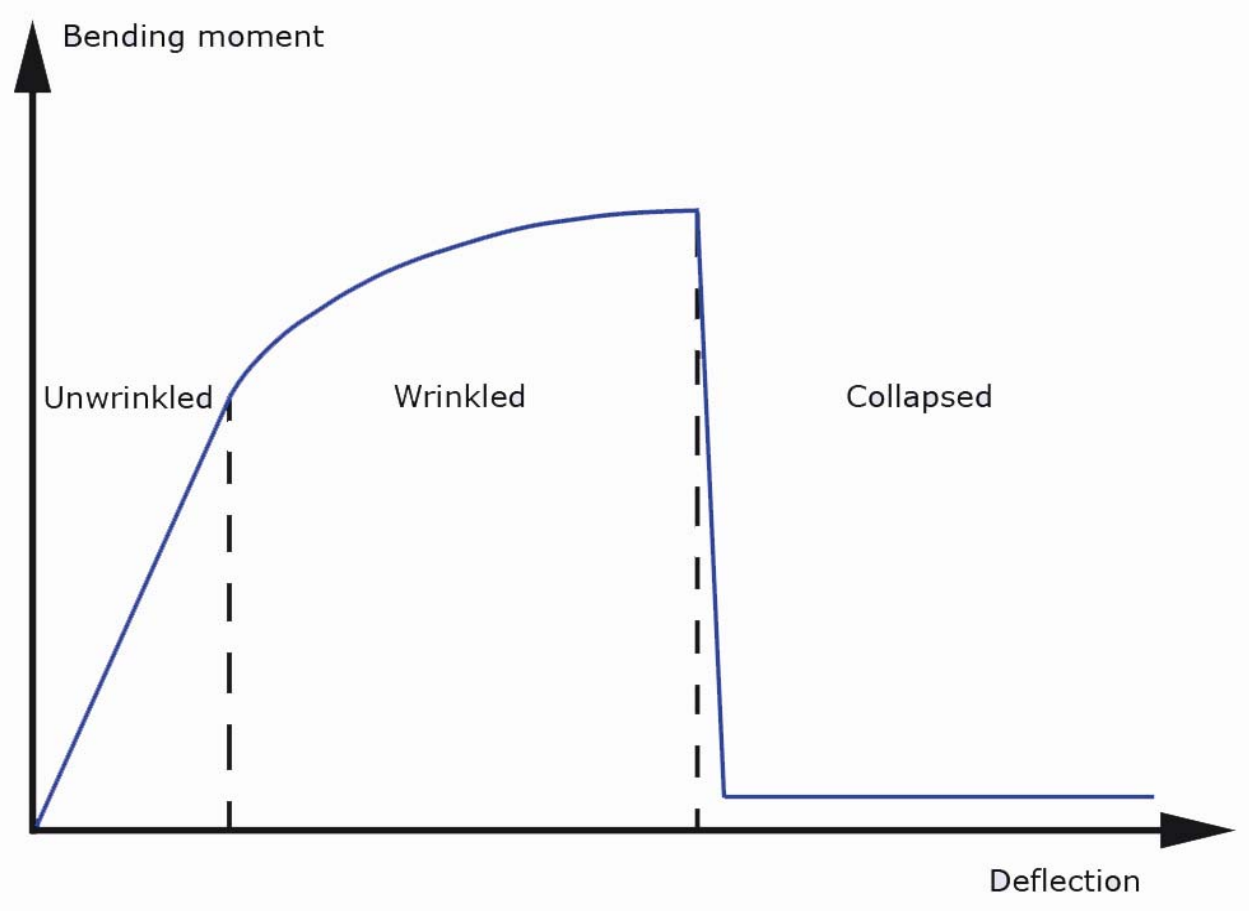

Figure 1, the three states of an inflatable beam

The applicability of the wrinkled state does pose a challenge in simulating the structure. As stated earlier, the wrinkled state is characterized by non-linearities and local effects influencing the behavior of the entire structure. In order to simulate such a structure, this paper proposes the approach through multi-body dynamics.

\section{The multi-body dynamics approach}

The state in which an inflatable structure finds itself is governed by geometry and internal pressure, as well as the externally applied loads. Modeling such a structure in conventional finite element packages results in very complex models which require significant system resources, and above all, time in order to be solved. According to Veldman [REF 17] the mesh density has a large influence on the results, especially when wrinkling occurs. Veldman proposes a model where the finest mesh is located where wrinkles will occur. The model which he analyzed is a 1 meter long cantilever beam which is loaded at the tip. Both straight and truncated beams are tested. Such a modeling approach is a possibility for simple models of a single beam where the externally applied load is fully known in advance. The location of the wrinkles is analytically obtainable. But for a complex structure, which can be loaded by many changing loads, the location of wrinkling constantly changes. Therefore, such a model would have to have a fine mesh everywhere, since the location of the wrinkles can simply not be anticipated beforehand. And so one would end up with a very demanding model.

For a designer of e.g. kites or boats, complicated finite element models coupled with long computation times on expensive computers form a hurdle which is not easily taken. Furthermore, Veldman [REF 17] showed that the modeling results of beams in a wrinkled state showed a significant discrepancy with analytical theory and experimental results. It is therefore concluded that finite element analysis of kite structures is a complicated and time-consuming process which doesn't always lead to satisfactory results. A designer preferes a faster modeling method which can be run on a conventional desktop computer. The designer is also not necessarily interested at 
exactly what angle and which location wrinkles occur. He is interested in the shape of the structure under different externally applied loads.

Consider a straight cantilever beam, as was modeled in [REF 17]. The beam is modeled as a number of rigid sections joint together using spherical joints. Figure 2 shows a graphical representation of such a model.

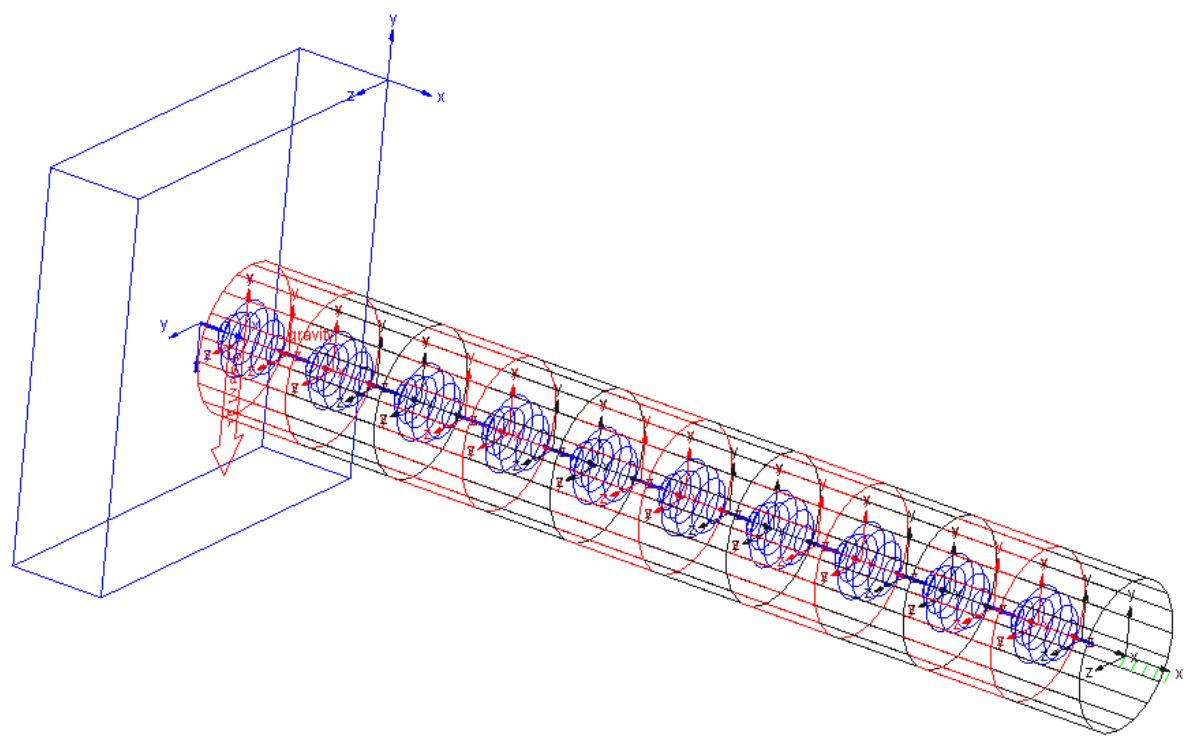

Figure 2, rigid cylindrical elements connected using spherical joints.

The spherical joints allow rotation along three axis. Two of these rotations can be considered as bending and one of these rotations can be considered as torsion. This beam is now nothing more than a chain of rigid bodies which exhibit no stiffness and no damping. The behavior of the structure is introduced by means of forces which act on the joints themselves. In the case of these tubular structures, its behavior is modeled by introducing a three dimensional torque vector on each of the joints. Figure 3 shows a close-up representation of the three dimensional torque vector.

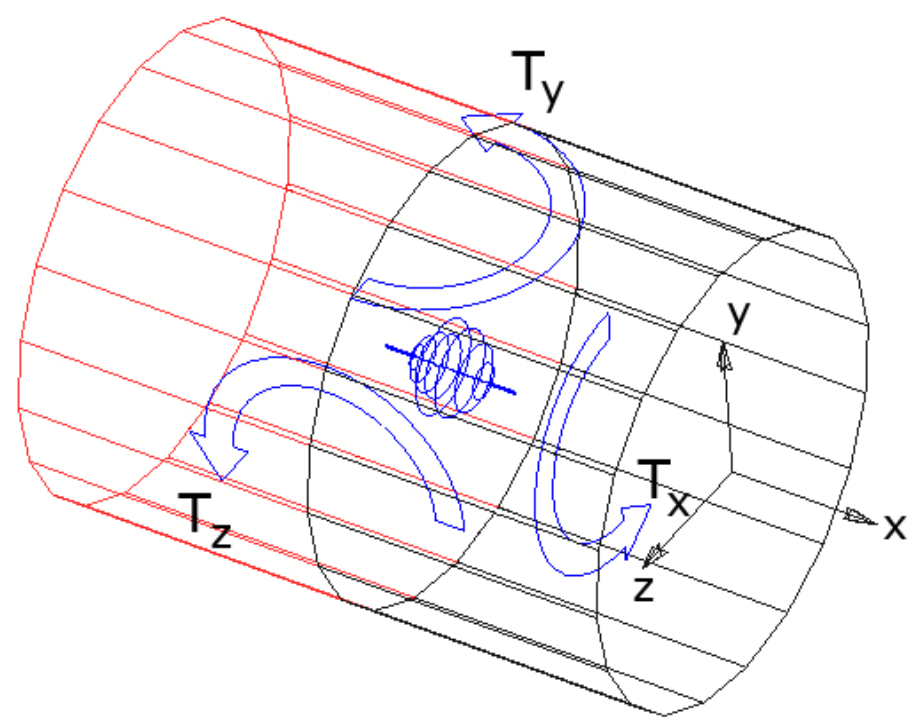

Figure 3, two rigid cylindrical elements joint together by a spherical joint. A 3-dimensional torque vector gives the joint its rigidity 
In figure $3, \mathrm{~T}_{\mathrm{y}}$ and $\mathrm{T}_{\mathrm{z}}$ are the torque vectors which govern the bending behavior of the beam. The torque vectors act as springs which give the beam its bending rigidity. $T_{x}$ is the torque vector which governs the torque of the beam. It acts as a torque spring, giving the beam its torsional stiffness. The stiffness of these springs determines the behavior of the inflatable beam. When the beam is in the unwrinkled state and both bending and torsion can be considered linear (for isotropic materials), the spring stiffness is a constant. In the wrinkled state, the stiffness of the springs starts to decrease with increasing load. Once the point of collapse is passed, the stiffness in the springs reduces to a value close to zero.

\section{Approximating the bending behavior}

The stiffness of torque vectors $T_{y}$ and $T_{z}$ determine the bending behavior of the beam. In order to simulate the bending as is depicted in figure 1 , the stiffness of the torque vectors $T_{y}$ and $T_{z}$ are dictated by a spline. The shape of this spline allows for a variable stiffness which is dependent on beam radius, internal pressure and externally applied loads. Figure 4 shows a bending spline which is used for simulation in MSC ADAMS.

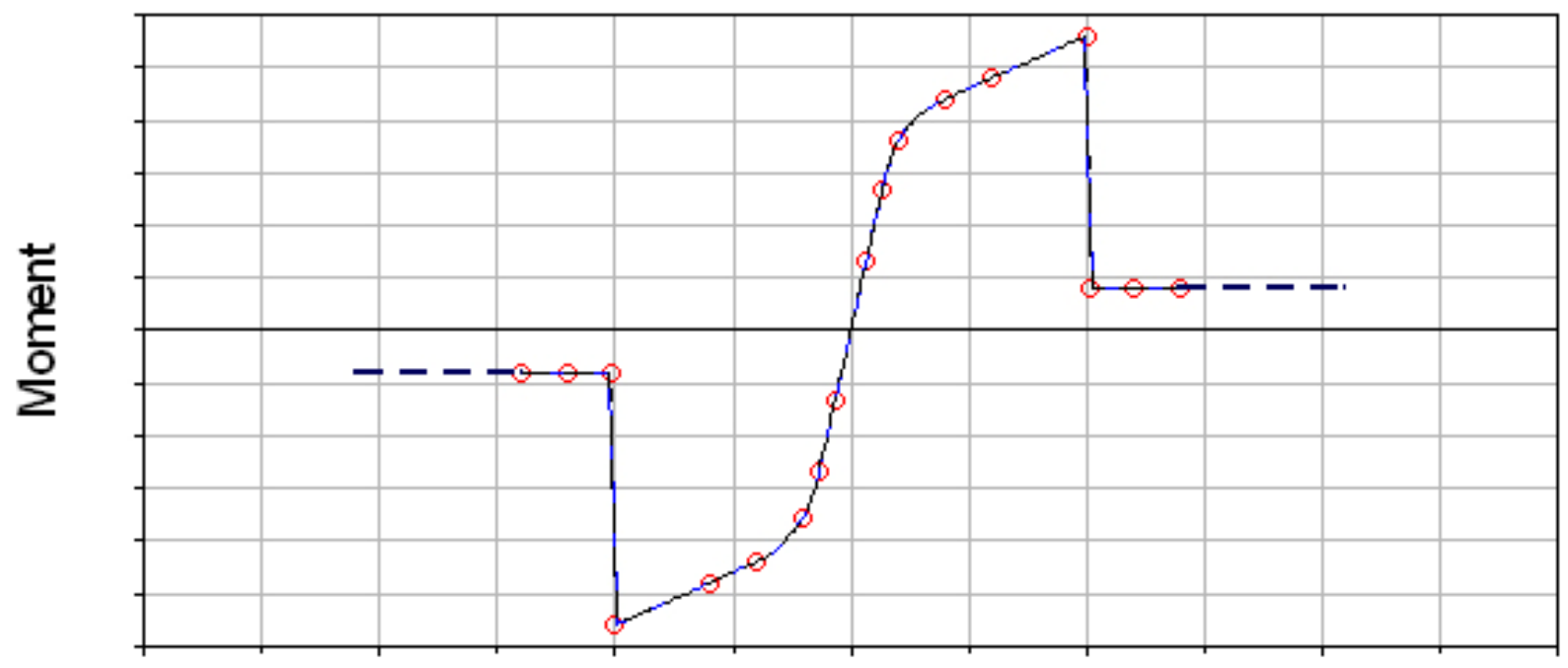

\section{Deflection}

\section{Figure 4, the spline which governs the bending behavior of the simulated inflatable tube.}

Each of the three states is governed by three pivot points, indicated in figure 4 by red circles. In the model, the bending and torsion angle at each of the spherical joints is constantly monitored by measures and is relayed to the local three dimensional torsion vector. The torsion vector uses these measures to determine its particular stiffness at that point in time by means of the spline in. The pivot points govern the shape of the spline and thereby the behavior of the beam under loading. In essence, there are three splines for each torque vector, governing its three degrees of freedom. In practice, the inflatable beams will be cylindrical and thus axi-symmetrical. This means that bending stiffness in $\mathrm{Y}$ and $\mathrm{Z}$ direction will share the same spline. The point where the spline crosses the horizontal axis is the point where the structure is under zero load. The spline is point-symmetrical in order to model both positive and negative bending moments. From the center point, the first three pivot points govern the unwrinkled state. Of these three pivot points, the top point indicates the moment at which wrinking first occurs. The second set of three pivot points govern the wrinkled state. In the wrinkled state, the bending behavior is no longer linear. The positioning of the three pivot points are able to curve the spline in such a way, that this non linear bending behavior is approximated. The last of these three pivot points is the point at which collapse occurs. The last three pivot points govern the collapsed behavior. The first of these points has a deflection value which is very close to the deflection value of the last pivot point in the wrinkled state. This is to simulate the sudden loss of rigidity of the structure. The small amount of residual rigidity, indicated by the small value of the last three pivot points is more a numerical issue than a physical one. By keeping the values non-zero, numerical problems within the simulation are avoided. 
In principle, the pivot points, and with it the splines, can differ for every joint. The placement of the pivot points is determined by the local radius of the beam and the internal pressure within. For a straight cantilever beam like depicted in figure 2, the bending splines will be equal at every joint. The beam has a constant radius and consists of a single envelope which means the pressure is the same over the entire length of the beam. This ensures that the pivot points are identical for every joint. For a tapered beam, however, the radius is a function of the axial coordinate. This means that the spline will differ for every joint. In such a beam, wrinkling does not necessarily occur at the root of the beam. The location of the first wrinkle is the result of a balance between local stiffness dictated by the local spline and the local bending moment.

\section{Obtaining the pivot points}

In order to relate the pivot points to the local beam radius and the internal pressure, the values for the pivot points are obtained through comparison of the model and experimental results. In [REF 3], bending tests were conducted on beams with differing internal pressures. These results are used to write scaling functions for the spline pivot points. These scaling functions have the form as indicated by equation 3 where A and B are dimensionless scaling factors which relate to a specific pivot point.

$$
y=A\left(p r^{3}\right)^{B}
$$

The bending tests in [REF 3] were conducted on 1 meter long inflatable tubes with a radius of 7.5 centimeters. A test rig was created where the beam was clamped on one end and loaded by a tip force on the other end. The data is used to determine the placement of the pivot points. This resulted in a simulation of the cantilever beam. Figure 5 shows the result of the measured values and the resulting simulation. This comparison is done on a beam with an internal overpressure of 0.2 bar.

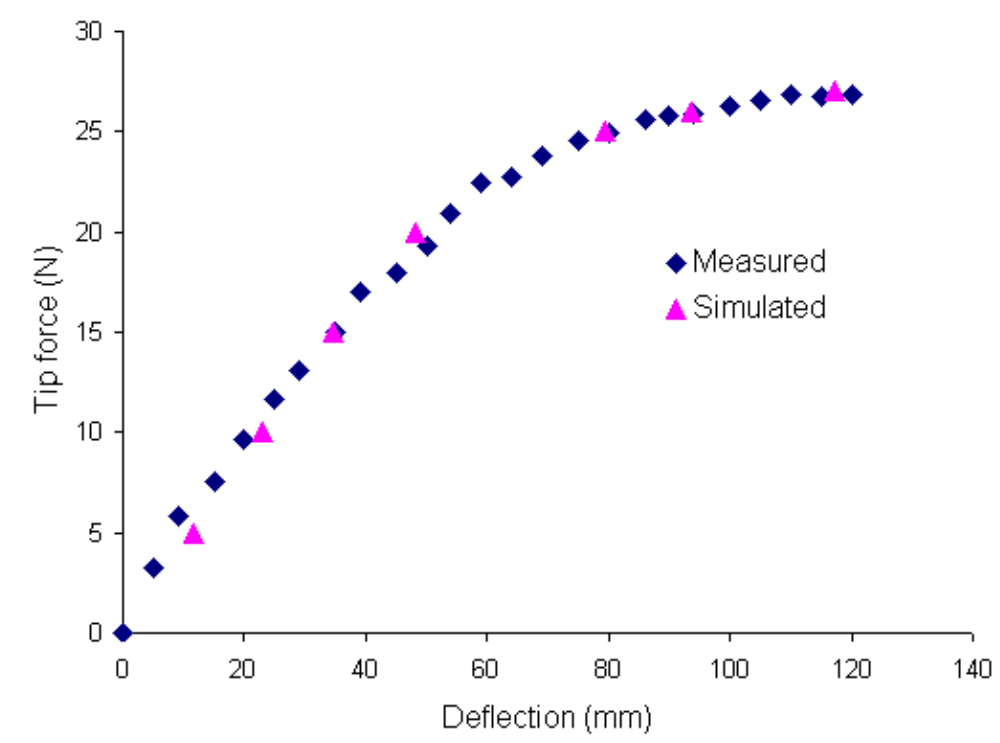

Figure 5, a comparison of measured and simulated (matched) values for a beam at 0.2 bar overpressure.

The simulation closely matches the measured values. This is to be expected because the values for the pivot points are manually tweaked so the two graphs match. What this graph shows is that a spline exists which enables the model to match the measured data. The same matching test was also performed for the data at 0.4 , and again, it was concluded that the correct spline to model this behavior exists.

The data from matching a spline to the existing measured curve is now used to write scaling functions for the pivot points. This allows a simulation of the beam at any internal overpressure and beam radius. These scaling functions are functions of internal pressure and beam radius and they create pivot points on which the bending spline is based. 
Figure 6 shows several bending splines created by using the scaling functions to create the pivot points. The triangle-shaped markers indicate the data matched to the measured values. It can be seen that the spline created by the pivot points which are obtained through the scaling functions closely match the pivot points obtained through matching of the measured values.

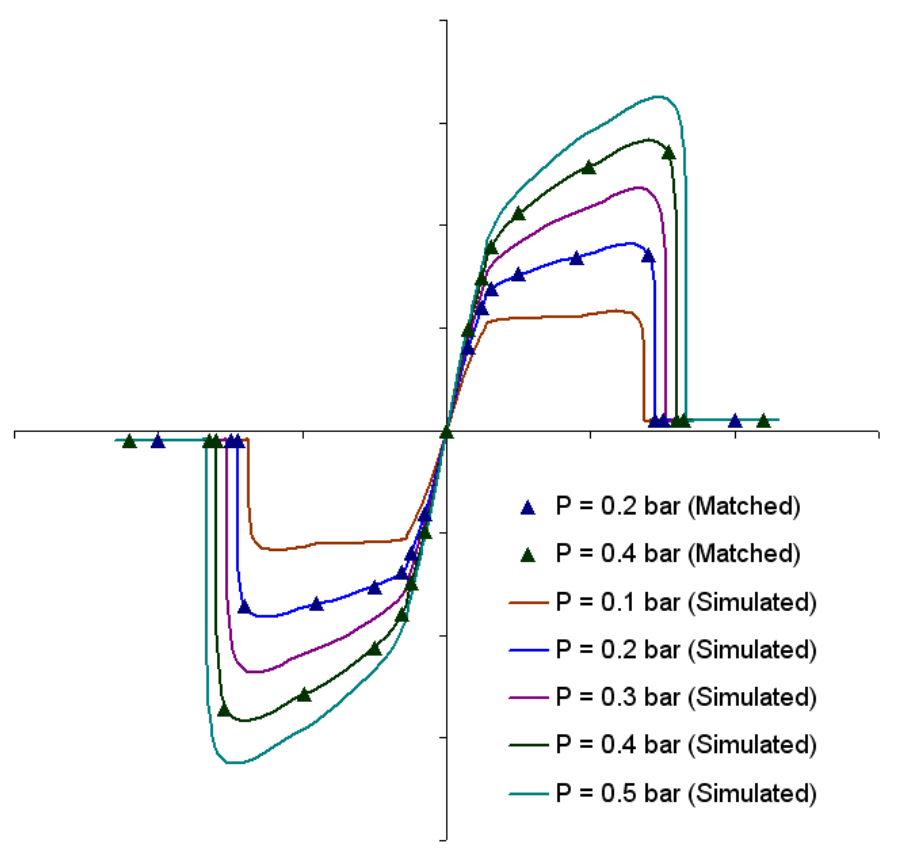

Figure 6, the matched and simulated splines.

\section{Simulation cases}

First, a simulation is done of the aforementioned cantilever beam. The scaling functions are used to create splines for beams with an internal pressure of 0.2 bar and 0.4 bar. Figure 7 shows a comparison between measured data (diamond-shaped markers) and simulated data (triangle shaped markers).

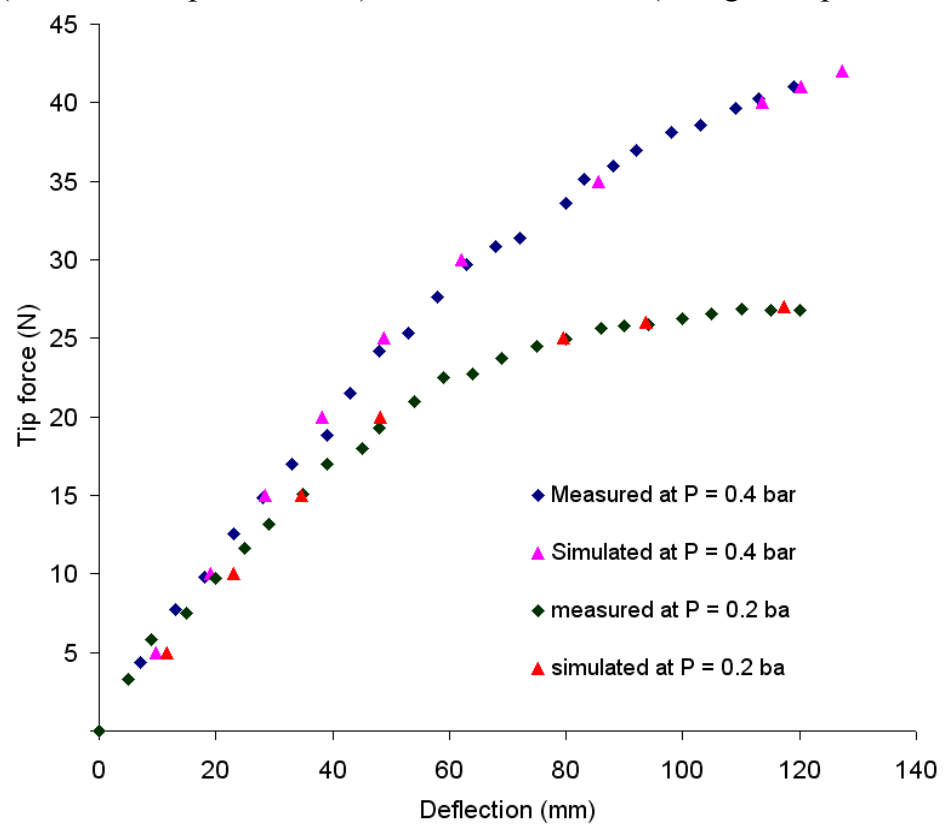

Figure 7, comparison between the measured and simulated data. 
From figure 7 it is concluded that the simulation is accurate in simulating a simple cantilever beam. Important to note is that the simulation runs real-time on a conventional desktop computer.

A more complex structure is that of a surf kite. The kite simulated here is called a tube kite, due to its arc-shaped inflatable leading edge tube. In order to validate the simulation using this tube kite, a static test is conducted and compared to simulated data. The kite is fixed at the wing tips and the arc is held upright. The kite is inflated to an overpressure of $0.25 \mathrm{bar}$. At the center of the arced wing, a load is applied and the deflection of the center point is measured. The inflatable tube is a double-tapered arc shaped beam consisting of a single envelope. Figure 8 shows the test setup and the simulated kite side-by-side.
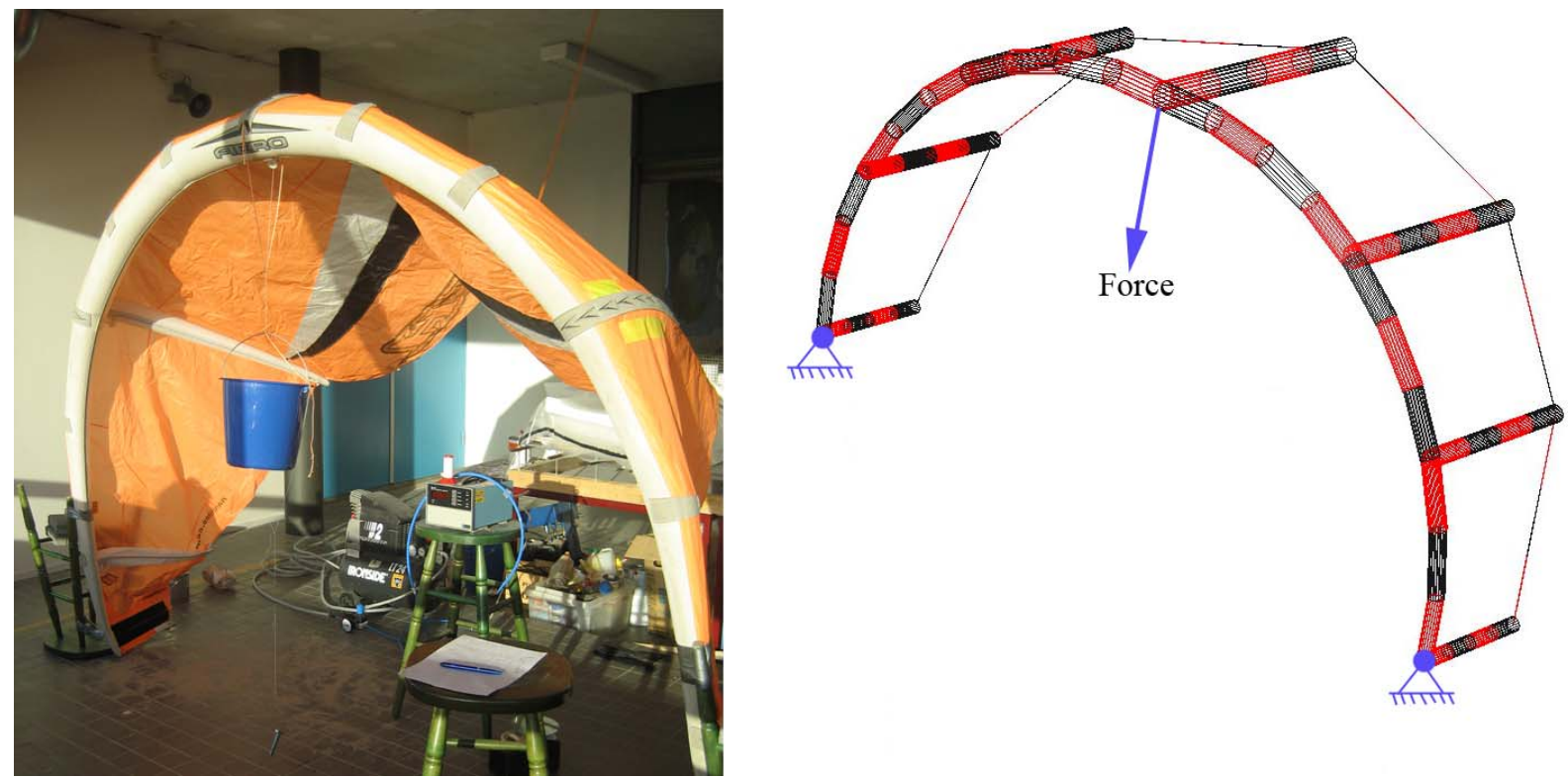

Figure 8, the test rig and the model side-by-side.

Figure 9 shows the result of the bending test. The Error bars indicate the margin for measurement errors.

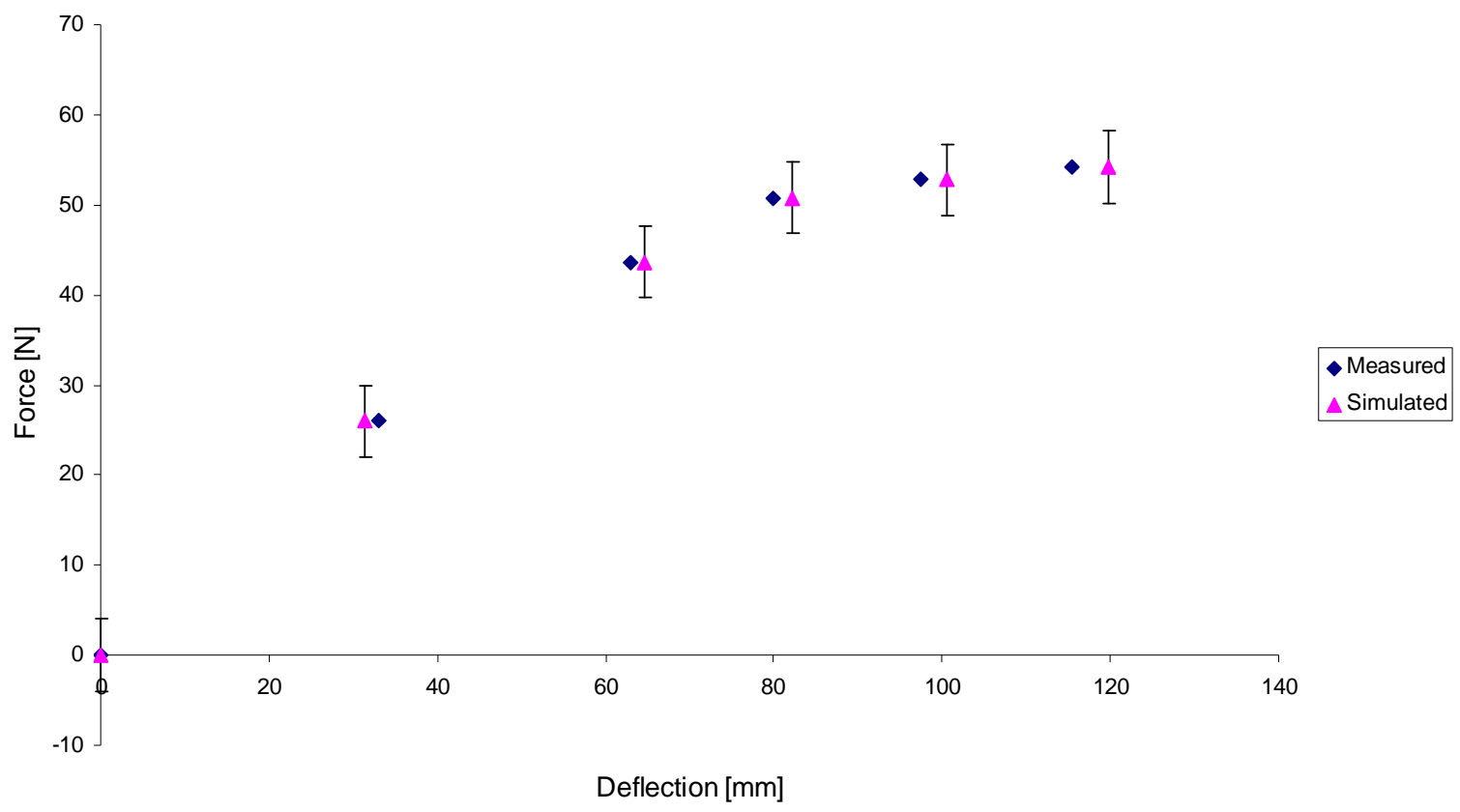

Figure 9, results of the bending test on the tube kite. 
Figure 9 shows a good correlation between the simulated and measured data. The simulated kite is a little less stiff. This may be caused by the fact that the tube in the kite has small reinforcements in the skin of the tube. Dacron tape is used to reinforce the seams, making the kite more durable. These reinforcements delay the occurrence of wrinkles and make the kite slightly stiffer than the simulated kite.

\section{Conclusions}

In this paper, it was shown that a multi-body dynamics approach to simulation of inflatable structures yields good results. In effect, very little actual mechanics of materials is used in this analysis. The simulation uses test data obtained from simple cases and extrapolates the results in order to simulate more complex structures. The simulation may be somewhat less accurate as complexity increases, but the computational resources needed for this simulation are significantly less than for a finite element model. As a design tool for e.g. kite builders, this simulation can give a good insight into the flexibility of an inflatable kite.

\section{Future research}

The work presented in this paper is a first effort to use a multi-body dynamics approach to simulating inflatable structures. Up to now, only bending behavior is analyzed. Future research must focus on torsion as well. Furthermore, the combination of bending and torsion is of great interest as well. In order to simulate e.g. inflatable wings a correct simulation of torsion en bending in an inflatable tube is a prerequisite.

In this paper, only point forces are introduced on the inflatable structure. In practice, distributed loads are more interesting due to the fact that most loads on an inflatable structure are, in fact, distributed loads. Wind loads on a pylon or snow loads on an inflatable shelter are examples of loads which are common. The simulation is perfectly able to deal with these loads as well. In the end, only the moment in the joints determine their deflection. Wether this moment is a result of a point load or a distributed load is of little interest. For wings, a particularly interesting load is the aerodynamic lift and drag. This load is dependent on velocity and angle of attack. Further research into these types of loads is required in order to realistically simulate a flying kite.

\section{Acknowledgments}

This work is part of the Laddermill project which is sponsored by the Delft University of Technology, the port of Rotterdam, the University of Groningen, the town of Delft, Gasunie, Fugro, Shell Research, Prolyte products, energy valley, the Town and Province of Groningen.

\section{References}

1. Comer, R.L. \& Levi, S. Deflections of an Inflated Circular Cylindrical Cantilever Beam. AIAA Journal, July 1963, $1,7$.

2. J.A. Main, S.W. Peterson, A.M. Strauss, beam-type bending of space-based inflatable structures, Journal of aerospace engineering, vol 8, nr 2, April 1995.

3. Breukels J., Design of a high altitude inflatable kite, Delft University of Technology, Faculty of Aerospace Engineering, August 2003.

4. Breukels J, Ockels, W.J., "Tethered wing design for the Laddermill project", WWEC 2005, Melbourne, Australia 5. Breukels J, Ockels, W.J., "Design of a large inflatable kiteplane" $48^{\text {th }}$ AIAA structures, structural dynamics and materials conference, Waikiki, Hawaii.

6. Breukels, J., Ockels, W.J., "a multi-body dynamics approach to a cable simulation for kites" IASTED AsiaMS2007, Beijing, China (to be published).

7. Veldman, S.L., Vermeeren, C.A.J.R., Bersee, H.E.N., Bergsma, O.K., Conceptual design of a high altitude kite, Faculty of Aerospace Engineering, Delft University of Technology, AIAA-2002-1735.

8. A. Adler, M. Mikulas, and J. Hedgepeth. Static and dynamic analysis of partially wrinkled membrane structures. In AIAA paper, AIAA-2000-1810. 2000. 
9. G. Brown, R. Haggard, and B. Norton. Inflatable structures for deployable wings. In AIAA conference paper, AIAA-2001-2068. 2001.

10. H. Chui, R. Benson, M. Fiscella, and S. Burns. Mechanical and thermal wrinkling of polymer membranes. journal of Applied Mechanics, pp. 67-70, 1994.

11. W. Fichter. a theory for inflated thin-wall cylindrical beams. Tech. Rep. TN D-3466, NASA, 1966.

12. W. Flugge. Stresses in shells. Springer verlag, 1966.

13. Nielsen, J.N., Theory of flexible aerodynamic surfaces, Journal of applied mechanics, September 1963.

14. S.L. Veldman, C.A.J.R. Vermeeren, O.K. Bergsma., Geometrical influences on the structural efficiency of an inflatable beam subjected to bending, ICAMM conference, 21-23 January 2003, Durban, South Africa.

15. S.L. Veldman, Load analysis of inflatable truncated cones, AIAA-2003-1827

16. Baker, E.H., Kovalevsky, L., Rish, F.L., Structural analysis of shells, McGraw-Hill Book Company, 1972 ISBN 0-07-003354-4

17. S. Veldman. "Design and analysis methodologies for inflated beams". Ph.D. thesis, Delft University of Technology, 2005.

18. C. Jenkins and J. Leonard. Dynamic wrinkling of visco-elastic membranes. Journal of Applied Mechanics, pp. $575\{582,1993$.

19. J. Main. Analysis and design of inflatable aerospace structures. Tech. rep., Vanderbilt University, 1993.

20. T. Murphey and M. Mikulas. Nonlinear e_ects of material wrinkles on the stiffness of thin polymer fims. In proceedings of the 40th AIAA/ASME/ASCE/AHS/ASC Structures, Structural

Dynamics, and Materials Conference and Exhibit, AIAA-1999-1341. 1999.

21. M. Stein and J. Hedgepeth. Analysis of partly wrinkled membranes. Tech. Rep. TN D-813, NASA, 1961.

22. S. Veldman and O. Bergsma. Designing inatable structures. In Proceedings of the IASS symposium on lightweight structures in civil engineering. 2002.

23. J. Webber. Deflections of inflated cylindrical cantilever beams subjected to bending and torsion. Aeronautical journal, pp. 306-312, 1982. 